\title{
Errata
}

\section{Erratum: Inflatonless inflation}

[Phys. Rev, D 52, 1895 (1995)]

A. Dobado and A. L. Maroto

PACS number(s): 98.80.Cq, 04.50. $+\mathrm{h}, 99.10 .+\mathrm{g}$

On page 1898, first column, Eq. (21) reads

$$
\mathcal{L}_{\text {eff }}=-\frac{M_{P}^{2}}{16 \pi} R+\frac{\alpha}{M_{P}^{2}} R_{\delta \gamma}^{\alpha \beta} R_{\delta \rho}^{\sigma \gamma} R_{\alpha \beta}^{\sigma \rho} .
$$

It should read

$$
\mathcal{L}_{\text {eff }}=-\frac{M_{P}^{2}}{16 \pi} R+\frac{\alpha}{M_{P}^{2}} R^{\alpha \beta}{ }_{\gamma \delta} R^{\gamma \delta}{ }_{\sigma \rho} R^{\alpha \beta}{ }_{\alpha \beta} .
$$

